\title{
Unidirectional lasing in semiconductor microring lasers at an exceptional point [Invited]
}

\author{
Stefano Longhi, ${ }^{1, *}$ and Liang Feng ${ }^{2}$ \\ ${ }^{1}$ Dipartimento di Fisica, Politecnico di Milano and Istituto di Fotonica e Nanotecnologie del Consiglio Nazionale delle Ricerche, \\ Piazza L. da Vinci 32, I-20133 Milano, Italy \\ ${ }^{2}$ Department of Electrical Engineering, The State University of New York at Buffalo, Buffalo, New York 14260, USA \\ *Corresponding author: longhi@fisi.polimi.it
}

Received 19 May 2017; revised 21 June 2017; accepted 23 June 2017; posted 23 June 2017; published 24 July 2017

Recent experiments demonstrated that chiral symmetry breaking at an exceptional point (EP) is a viable route to achieve unidirectional laser emission in microring lasers. By a detailed semiconductor laser rate equation model, we show here that unidirectional laser emission at an EP is a robust regime. Slight deviations from the EP condition can break preferential unidirectional lasing near threshold via a Hopf instability. However, above a "second" laser threshold, unidirectional emission is restored.

\section{INTRODUCTION}

Microdisk/microring lasers are attracting continuous and increasing attention as key elements of future photonic integrated circuits [1-4]. Such lasers can support clockwise (cw) and counterclockwise (ccw) traveling-wave modes with chiral symmetry. In nongyromagnetic materials, they are degenerate because of time-reversal symmetry, and spurious feedback in the cavity couples them [5]. As a result, the emission of an active microring cavity with time-reversal symmetry is usually not unidirectional, and different dynamical regimes can be observed, which arise from linear and nonlinear mode coupling [6-9].

Several works have demonstrated that unidirectional laser emission can be forced by breaking chiral symmetry [10-21] without resorting to nonreciprocal elements, which are challenging to realize at the microscale and the nanoscale. A viable route to obtain chiral modes and unidirectional laser emission is to introduce asymmetric couplings between $\mathrm{cw}$ and $\mathrm{ccw}$ modes, as originally suggested in Ref. [11]. Under asymmetric coupling, the mode circulating in the direction that receives more cross coupling is the preferred lasing mode [11]. Interestingly, when one of the two couplings vanishes (unidirectional coupling), one operates at the so-called exceptional point (EP) $[22,23]$, where maximal chirality is obtained and complete unidirectional laser emission is observed [19-21]. Partial or complete directional lasing based on EP operation in microlasers has been demonstrated in a few recent works by use of two Rayleigh scatterers $[19,20]$ or by combined index and loss gratings [21]. The prediction of perfect unidirectional emission at an EP is based on the chiral nature of the linear resonator modes at the EP $[19,20,23]$. However, deviations from exact EP operation as well as the impact of nonlinear mode coupling on directional laser emission above threshold have been mostly eluded so far. Some preliminary numerical results based on a laser rate equation model, reported in an earlier work [21], indicate that unidirectional lasing is expected to be stable near the EP operation. However, some instability may arise when the microring design deviates from exact EP operation.

In this work, we extend our preliminary numerical results of Ref. [21] and provide a more comprehensive theoretical study of the impact of nonlinear mode coupling in the dynamical behavior of the EP-based semiconductor microring laser. Our theoretical study explains the origin of instability and provides general laser design criteria for stable unidirectional mode operation. By means of a phase analysis of semiconductor laser rate equations based on asymptotic methods (not reported in previous work [21]), we can analytically show by linear stability analysis that at exact EP operation, unidirectional laser emission is always a stable regime above threshold. Near EP operation, the phase analysis shows that deviations from exact EP operation can destabilize unidirectional emission near threshold via a Hopf instability. However, above a second laser threshold, preferential continuous-wave unidirectional lasing is restored. The phase analysis also shows that, as expected [6,7], chiral symmetry and bistable laser output in either one of the two counterpropagating traveling-wave modes (TWMs) occur at high driving currents as a result of nonlinear mode dynamics. 


\section{MICRORING LASER AT EP: RATE-EQUATION MODEL AND PHASE ANALYSIS}

\section{A. Semiconductor Laser Rate Equations}

The semiconductor laser rate equations that describe the dynamics of two counterpropagating TWMs in a ring cavity read as $[6,7,24,25]$ [Fig. 1(a)]

$$
\begin{aligned}
\frac{\mathrm{d} E_{1}}{\mathrm{~d} t}= & \kappa(1+i \alpha)\left[N\left(1-s\left|E_{1}\right|^{2}-c\left|E_{2}\right|^{2}\right)-1\right] E_{1} \\
& +i \kappa_{1} E_{2}
\end{aligned}
$$

$$
\begin{aligned}
\frac{\mathrm{d} E_{2}}{\mathrm{~d} t}= & \kappa(1+i \alpha)\left[N\left(1-s\left|E_{2}\right|^{2}-c\left|E_{1}\right|^{2}\right)-1\right] E_{2} \\
& +i \kappa_{2} \exp (i \Theta) E_{1}
\end{aligned}
$$

$$
\begin{aligned}
\frac{\mathrm{d} N}{\mathrm{~d} t}= & \gamma\left[\mu-N-N\left(1-s\left|E_{1}\right|^{2}-c\left|E_{2}\right|^{2}\right)\left|E_{1}\right|^{2}\right. \\
& \left.-N\left(1-s\left|E_{2}\right|^{2}-c\left|E_{1}\right|^{2}\right)\left|E_{2}\right|^{2}\right],
\end{aligned}
$$

where $E_{1,2}(t)$ are the slowly varying normalized amplitudes of $\mathrm{cw}$ and ccw TWMs, respectively, $N(t)$ is the carrier density, $\kappa$ is the field decay rate, $\gamma$ is the decay rate of the carrier population, $\alpha$ is the linewidth enhancement factor, and $\mu$ is the normalized injection current ( $\mu \sim 0$ at transparency, $\mu \sim 1$ at lasing threshold). The two counterpropagating modes interact both in a linear and nonlinear fashion. Linear mode coupling is described by the coupling strengths $\kappa_{1,2}>0$ and the relative phase $\Theta$ of the coupling. For reactive (Hermitian) coupling, $\kappa_{1}=\kappa_{2}$ and $\Theta=0$. In a general non-Hermitian coupling scheme, $\kappa_{1}, \kappa_{2}$, and $\Theta$ can take arbitrary values, with the EP condition corresponding to $\kappa_{2}=0$. In experiments, control of $\kappa_{1}, \kappa_{2}$, and $\Theta$ in a microring can be realized by the use of two Rayleigh scatterers $[19,20]$ [Fig. 1(b)] or by a combination of phase-shifted index and loss gratings [21] [Fig. 1(c)]. Here we will focus our attention on laser operation near an EP, with a vanishing or small value of coupling $\kappa_{2}$ as compared to $\kappa_{1}$. Nonlinear mode coupling, arising from self- and cross-saturation effects, is described by self- and cross-saturation parameters $s$ and $c$. In a typical semiconductor laser, the self-and cross-saturation parameters satisfy the condition $c=2 s$ [6-9], and therefore we will typically consider the case $c=2 s$. However, as shown in previous works [7], the qualitative dynamical behavior of semiconductor ring lasers does not depend on the specific values of $s$ and $c$, provided that the condition $c>s$ is fulfilled. Such a point will be also clear by the phase analysis of laser rate Eqs. (1)-(3) presented in the next subsection. (a)

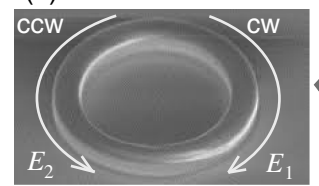

(b)

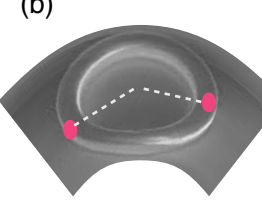

(c)

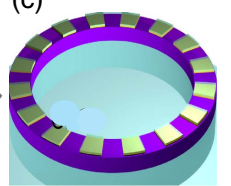

Fig. 1. (a) Schematic of a microring sustaining $\mathrm{cw}$ and $\mathrm{ccw}$ TWMs. Control of linear mode couplings $\kappa_{1,2}$ based on the use of (b) two Rayleigh scatterers (dots) [19,20], and (c) combined phase-shifted index and loss gratings [21].

\section{B. Phase Analysis}

Preliminary numerical results of the laser rate Eqs. (1)-(3) reported in the Supplementary Material of our previous work [21] showed that unidirectional laser emission at an EP is a stable regime well above threshold. However, an oscillatory instability near threshold is observed when considering deviations from exact EP operation. To provide some analytical and physical insights into the laser dynamics near the EP observed in full numerical simulations, we present here a phase analysis of the laser rate Eqs. (1)-(3) using asymptotic methods in the limit $\kappa / \gamma \rightarrow \infty$, which generally applies to semiconductor laser dynamics $[24,25]$. The phase analysis explains the onset of a Hopf instability for slight deviations from EP operation, restoration of stable unidirectional emission above a second laser threshold, and bistable laser emission in either one of the two TWMs at high current levels as a result of nonlinear mode competition. The asymmetric coupling regime $\kappa_{1} \neq \kappa_{2}$, previously investigated in Ref. [25], gives rise to multistable and excitable laser behavior. Here we mainly focus on the operational regime close to the EP operation, where we show that unidirectional emission is a stable regime, and derive some analytical results on the second laser threshold and Hopf instability boundaries, which were not derived in previous works and are of major relevance for the design of stable unidirectional operation of the semiconductor microring laser.

In the limit $\kappa / \gamma \rightarrow \infty$, an asymptotic analysis of Eqs. (1)-(3) in the large parameter $\kappa / \gamma$ shows that the carrier density $N$ remains almost clamped to the saturated value $N \simeq 1$, whereas the total intensity $\left|E_{1}\right|^{2}+\left|E_{2}\right|^{2}=\mu-1$ does not change with time [24]. After setting

$$
\begin{aligned}
& E_{1}=\sqrt{\mu-1} \cos \left(\frac{\theta}{2}+\frac{\pi}{4}\right) \exp \left(i \phi_{1}\right), \\
& E_{2}=\sqrt{\mu-1} \sin \left(\frac{\theta}{2}+\frac{\pi}{4}\right) \exp \left(i \phi_{2}\right),
\end{aligned}
$$

the following coupled equations for the phases $\theta(t)$ and $\psi(t) \equiv \phi_{2}-\phi_{1}$ can be derived from Eqs. (1)-(3):

$$
\begin{aligned}
\frac{\mathrm{d} \theta}{\mathrm{d} t}= & J \kappa_{1} \sin \theta \cos \theta+(1-\sin \theta)\left[\kappa_{2} \sin (\psi-\Theta)-\kappa_{1} \sin \psi\right] \\
& +2 \kappa_{1} \sin \psi,
\end{aligned}
$$

$$
\begin{aligned}
\frac{\mathrm{d} \psi}{\mathrm{d} t}= & \alpha J \kappa_{1} \sin \theta+\kappa_{2} \operatorname{cotg}\left(\frac{\theta+\pi / 2}{2}\right) \cos (\psi-\Theta) \\
& -\kappa_{1} \operatorname{tg}\left(\frac{\theta+\pi / 2}{2}\right) \cos \psi
\end{aligned}
$$

where

$$
J \equiv \frac{\kappa}{\kappa_{1}} c(\mu-1)\left(1-\frac{s}{c}\right)
$$

is the normalized pump parameter ( $J=0$ at threshold). Note that within the phase analysis approximation, the laser dynamics depend on the dimensionless parameters $J \geq 0, \kappa_{2} / \kappa_{1} \geq 0$, $\Theta$, and $\alpha$ solely. Note also that within the limits of validity of the phase analysis, the laser dynamics are qualitatively independent of the values of self- and cross-saturation parameters 
provided that the condition $c>s$ is satisfied. In fact, according to Eq. (8), a change of the ratio $c / s$ just corresponds to a renormalization of the pump parameter $J$.

\section{STABLE UNIDIRECTIONAL LASER EMISSION AT EXACT EP OPERATION}

As a first application of the phase analysis, let us analytically prove that, at exact EP operation, unidirectional laser emission is always a stable operational regime above the laser threshold. In fact, at exact EP operation, one has $\kappa_{2}=0$ and Eqs. (6) and (7) simplify as follows:

$$
\begin{aligned}
& \frac{\mathrm{d} \theta}{\mathrm{d} t}=J \kappa_{1} \sin \theta \cos \theta+\kappa_{1}(1+\sin \theta) \sin \psi, \\
& \frac{\mathrm{d} \psi}{\mathrm{d} t}=\alpha J \kappa_{1} \sin \theta-\kappa_{1} \operatorname{tg}\left(\frac{\theta+\pi / 2}{2}\right) \cos \psi .
\end{aligned}
$$

The solutions to Eqs. (9) and (10), corresponding to continuous-wave unidirectional laser emission in the $\mathrm{cw}$ mode $\left(E_{2}=0,\left|E_{1}\right|^{2}=\mu-1\right)$, are given by

$$
\theta(t)=-\pi / 2, \quad \psi(t)=-\alpha \kappa_{1} J t .
$$

The stability of such solutions for any normalized pump parameter $J>0$ can be readily demonstrated by standard linear stability analysis. By letting $\psi(t)=-\alpha \kappa_{1} J t+\delta \psi(t)$ and $\theta(t)=-\pi / 2+\delta \theta(t)$, the linearized equations for the perturbations $\delta \psi(t)$ and $\delta \theta(t)$ read as

$$
\begin{aligned}
\frac{\mathrm{d} \delta \psi(t)}{\mathrm{d} t} & =-\frac{\kappa_{1}}{2} \delta \theta(t) \cos \left(\alpha \kappa_{1} t\right), \\
\frac{\mathrm{d} \delta \theta(t)}{\mathrm{d} t} & =-\kappa_{1} J \delta \theta,
\end{aligned}
$$

which yield damped solutions $\delta \psi(t), \delta \theta(t) \rightarrow 0$ for perturbations as $t \rightarrow \infty$. Such a result demonstrates that the unidirectional laser emission at an EP is a stable regime, even when nonlinear mode coupling above threshold is properly taken into consideration.

Besides the stationary $\mathrm{cw}$ unidirectional solution, other stationary solutions can be found by letting $\mathrm{d} \theta / \mathrm{d} t=0$ in Eqs. (9) and (10). Such solutions do not correspond to unidirectional emission and are a signature of multistable laser behavior $[8,9]$. The diagram of stationary solutions versus $J$ for a typical value of the linewidth enhancement factor $\alpha=3$ is shown in Fig. 2. Solid curves refer to stable stationary solutions, whereas dotted curves correspond to unstable solutions. Note that for $J<J_{1}$ with $J_{1} \simeq 1.05$, the unidirectional cw mode is the only stable solution, whereas for $J>J_{1}$, bistability is observed between $\mathrm{cw}$ and ccw laser emission. Note that, according to Eqs. (9) and (10), the pump parameter threshold $J_{1}$ at which bistability appears is a function of the linewidth enhancement factor $\alpha$ solely. It can be calculated in a closed form by considering the steady-state solutions to Eqs. (9) and (10) with $\theta \neq-\pi / 2$ and imposing $(\mathrm{d} J / \mathrm{d} \theta=0)$. After some calculations, one obtains

$$
J_{1} \simeq \frac{3.33}{\sqrt{1+\alpha^{2}}} .
$$

Note that $J_{1}$ decreases as $\alpha$ increases.

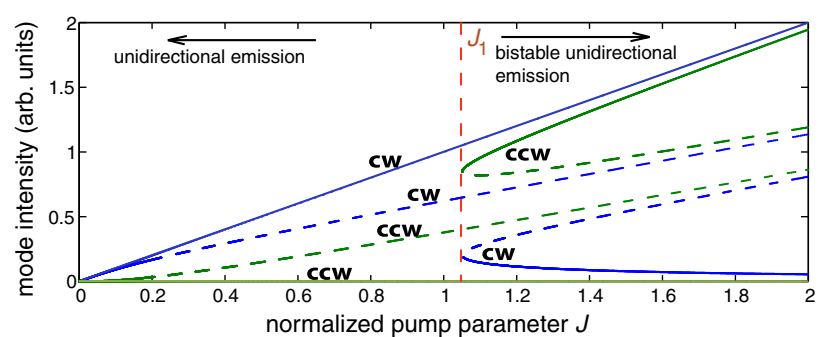

Fig. 2. Intensity of $\mathrm{cw}$ and $\mathrm{ccw}$ ring modes versus normalized pump parameter $J$ at an EP $\left(\kappa_{2} / \kappa_{1}=0\right)$ for $\alpha=3$. Solid and dashed curves refer to stable and unstable branches of stationary solutions, respectively. For $J<J_{1}$, the only stable solution corresponds to unidirectional laser emission in the $\mathrm{cw}$ mode $\left(E_{2}=0\right)$, whereas for $J>J_{1}$, bistable unidirectional emission, with either dominant $\mathrm{cw}$ or ccw modes, is observed. The value of $J_{1}$ depends on the linewidth enhancement factor $\alpha$ solely according to Eq. (14).

From a physical viewpoint, the appearance of bistable behavior at high pump levels $J>J_{1}$ means that the chiral symmetry of the microring is restored and laser emission can switch randomly into either one of the $\mathrm{cw}$ or ccw TWM by noise or external perturbations. Such a restoration of the chiral symmetry well above threshold, when the laser operates in the highly nonlinear regime and the linear coupling $\kappa_{1}$ works as a weak term in the dynamics, is in agreement with previous studies of mode competition in ring lasers $[6,7,26,27]$. According to Eqs. (8) and (14), the injection current level $\mu$ above which the bistable regime arises increases as the ratio $\kappa_{1} / \kappa$ increases. Therefore, to avoid bistable emission, a relatively strong and controllable coupling $\kappa_{1}$ is needed, like in the distributed feedback microring with two phase-shifted index and loss gratings [21]. Other methods, such as the use of two Rayleigh scatterers, generally yield low couplings $\kappa_{1}$, and thus the breakdown of unidirectional emission could be observed at relatively low injection currents above threshold. For example, assuming $c=2 s=0.01, \alpha=3$, and $\kappa \simeq 400 \mathrm{~ns}^{-1}$, which are typical values for parameter range variation in semiconductor ring lasers $[6-8,24]$, a coupling $\kappa_{1}$ larger than $7.6 \mathrm{~ns}^{-1}$ is required to avoid bistable emission for a pump parameter of $\mu<5$, i.e., for a current level up to five times its threshold value.

\section{DEVIATION FROM EP OPERATION: HOPF INSTABILITY AND THE SECOND LASER THRESHOLD}

In a practical design, the control of the couplings $\kappa_{1}$ and $\kappa_{2}$ is not perfect and slight deviations in the EP regime can be expected. One of the main theoretical predictions based on the phase analysis developed in Section 2.B is that for a nonvanishing value of $\kappa_{2} / \kappa_{1}$, unidirectional emission in the $\mathrm{CW}$ TWM can be destabilized close to threshold via a Hopf bifurcation. The most adverse case is observed for a phase of $\Theta=0$, and therefore we will limit to consider such a case. A typical example of the laser behavior for a nonvanishing value of $\kappa_{2} / \kappa_{1}$ is shown in Fig. 3. The figure depicts the diagrams of stable continuouswave laser emission for $\alpha=3, \Theta=0$, and $\kappa_{2} / \kappa_{1}=0.5$, i.e., for a considerable deviation from the exact EP operation. 


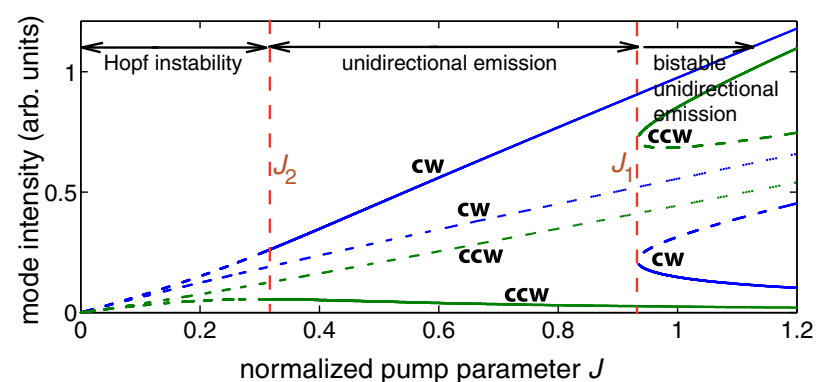

Fig. 3. Same as Fig. 2 but for $\kappa_{2} / \kappa_{1}=0.5, \Theta=0$, and $\alpha=3$. For $J<J_{2} \simeq 0.33$, all stationary solutions are unstable and the unidirectional TWM emission is destabilized by a Hopf instability. Laser emission in the dominant $\mathrm{cw}$ mode is realized in the pump parameter range of $J_{2}<J<J_{1}$.

As one can see, the stationary solution is destabilized near threshold for $J<J_{2}$, with $J_{2} \simeq 0.33$ for the case of Fig. 3 . In the range of $0<J<J_{2}$ the laser emission is typically oscillatory with nonstationary emission in $\mathrm{cw}$ and ccw TWMs; to observe stationary emission with the dominant cw TWM, the driven current should be increased above the threshold value $(J=0)$ such that $J>J_{2} \cdot J_{2}$ can be thus referred to as the "second" laser threshold, i.e., the threshold for stable laser emission in the dominant cw mode. For $J_{2}<J<J_{1}$, Fig. 3 shows that the laser emission is stationary with the $\mathrm{cw}$ mode being the dominant mode. However, deviation from exact EP operation leads to a nonnegligible excitation of the ccw mode as well. The deviation from perfect unidirectional emission can be measured by the directionality parameter $\mathcal{D}$, which is defined as

$$
\mathcal{D}=\frac{\left|E_{1}\right|^{2}-\left|E_{2}\right|^{2}}{\left|E_{1}\right|^{2}+\left|E_{2}\right|^{2}},
$$

with $\mathcal{D} \leq 1$ and $\mathcal{D}=1$ for perfect unidirectional emission in the $\mathrm{cw}$ mode. Within the phase approximation of the semiconductor laser rate equations, from Eqs. (4), (5), and (15), one readily obtains a simple expression of the directionality as a function of the phase $\theta(t)$

$$
\mathcal{D}=-\sin \theta .
$$

The directionality can be thus computed from the steady-state solutions of Eqs. (6) and (7). The directionality smoothly decreases as $\kappa_{2} / \kappa_{1}$ increases, starting from $\mathcal{D}=1$ at the exact EP operation $(\theta=-\pi / 2)$. Figure 4 shows the behavior of the directionality $\mathcal{D}$ versus $\kappa_{2} / \kappa_{1}$ for $\alpha=3$ close to the second laser threshold, i.e., for the normalized pump parameter $J=J_{2}^{+}$. As expected, the directionality is degraded as $\kappa_{2} / \kappa_{1}$ increases; a directionality larger than 0.9 is required to keep $\kappa_{2} / \kappa_{1}$ smaller than 0.065 . Similar behavior of directionality versus $\kappa_{2} / \kappa_{1}$ is obtained for other values of the linewidth enhancement factors.

According to the phase analysis, the second laser threshold $J_{2}$ is a function of $\kappa_{2} / \kappa_{1}$ and $\alpha$ solely, with $J_{2} \rightarrow 0$ as $\kappa_{2} / \kappa_{1} \rightarrow 0$, i.e., at exact EP operation. Figure 5 shows the numerically computed behavior of the second laser threshold $J_{2}$ versus $\kappa_{2} / \kappa_{1}$ and of the bistability boundary $J_{1}$ for a few values of the linewidth enhancement factor $\alpha$. At $J<J_{2}$, the stationary solution corresponding to the dominant $\mathrm{cw}$ mode is

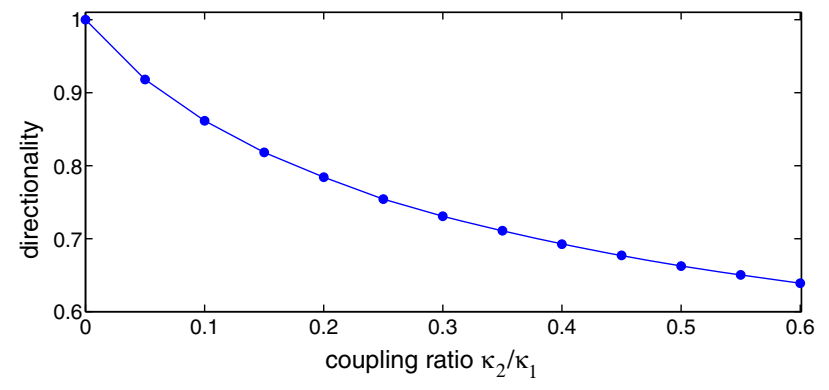

Fig. 4. Behavior of the directionality $\mathcal{D}$ versus coupling ratio $\kappa_{2} / \kappa_{1}$ for a linewidth enhancement factor of $\alpha=3$. The directionality is calculated close to the second laser threshold, i.e., for $J=J_{2}^{+}$.

destabilized via a Hopf bifurcation, whereas for $J>J_{1}$, the laser can switch into the other stable solution corresponding to bistable emission in almost $\mathrm{cw}$ or ccw modes. The normalized pump parameter $J_{1}$ of the bistability boundary is always larger than the normalized pump parameter $J_{2}$ of the second laser threshold, and its value slightly decreases from the one predicted by Eq. (14) as $\kappa_{2} / \kappa_{1}$ increases above zero. An analysis of the phase equations indicates that $J_{1} \rightarrow J_{2}^{+}$as $\kappa_{2} / \kappa_{1} \rightarrow 1^{-}$, i.e., when mode coupling becomes symmetric - in this limit, the stability domain of dominant $\mathrm{cw}$ mode laser emission, corresponding to the range $\left(J_{2}, J_{1}\right)$ of the normalized pump parameter, shrinks and chiral symmetry is fully restored.

The instability of the dominant $\mathrm{cw}$ mode solution for $J<J_{2}$ is a Hopf (oscillatory) instability, as one can analytically prove by a standard linear stability analysis of Eqs. (6) and (7). Let $\theta_{0}, \psi_{0}$ be the stationary solutions to Eqs. (6) and (7), corresponding to laser oscillation in the dominant cw stationary mode; the values of $\theta_{0}$ and $\psi_{0}$ can be obtained as the roots of transcendental equations, in which $\psi_{0}$ and $J$ are parametrized by $\theta_{0}$ as follows:

$$
\operatorname{tg} \psi_{0}=\frac{\cos \theta_{0}\left[\kappa_{2} \operatorname{cotg}\left(\frac{\theta_{0}}{2}+\frac{\pi}{4}\right)-\kappa_{1} \operatorname{tg}\left(\frac{\theta_{0}}{2}+\frac{\pi}{4}\right)\right]}{2 \alpha \kappa_{1}-\alpha\left(\kappa_{1}-\kappa_{2}\right)\left(1-\sin \theta_{0}\right)},
$$

$$
J=\frac{\left(\kappa_{1}-\kappa_{2}\right)\left(1-\sin \theta_{0}\right) \sin \psi_{0}-2 \kappa_{1} \sin \psi_{0}}{\sin \theta_{0} \cos \theta_{0}} .
$$

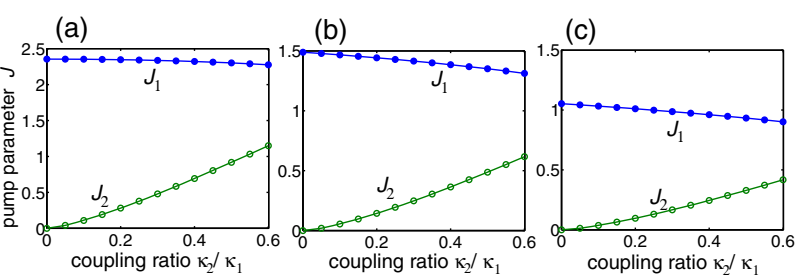

Fig. 5. Numerically computed behavior of the normalized pump parameter $J_{1}$, corresponding to the bistability boundary (upper curves, filled circles), and of $J_{2}$, corresponding to the "second" laser threshold (Hopf instability boundary, open circles), versus $\kappa_{2} / \kappa_{1}$ for $\Theta=0$ and for a few values of the linewidth enhancement factor. (a) $\alpha=1$, (b) $\alpha=2$, and (c) $\alpha=3$. The region embedded between the upper and lower curves corresponds to stable laser emission in the dominant $\mathrm{cw}$ mode. The lower and upper curves touch at $\kappa_{2} / \kappa_{1}=1$ (not shown in the figures). 
Small perturbations $\delta \theta(t)$ and $\delta \psi(t)$ around the steady-state solution evolve according to the linearized system

$$
\frac{\mathrm{d}}{\mathrm{d} t}\left(\begin{array}{c}
\delta \theta \\
\delta \psi
\end{array}\right)=\left(\begin{array}{ll}
\mathcal{M}_{11} & \mathcal{M}_{12} \\
\mathcal{M}_{21} & \mathcal{M}_{22}
\end{array}\right)\left(\begin{array}{c}
\delta \theta \\
\delta \psi
\end{array}\right),
$$

where the elements of the stability matrix $\mathcal{M}$ are given by

$$
\begin{aligned}
\mathcal{M}_{11}= & J \cos \left(2 \theta_{0}\right)-\left(\kappa_{2}-\kappa_{1}\right) \cos \theta_{0} \sin \psi_{0}, \\
\mathcal{M}_{12}= & \left(\kappa_{2}-\kappa_{1}\right)\left(1-\sin \theta_{0}\right) \cos \psi_{0}+2 \kappa_{1} \cos \psi_{0}, \\
\mathcal{M}_{21}= & \alpha J \cos \theta_{0}-\frac{\kappa_{2} \cos \psi_{0}}{2 \sin ^{2}\left(\frac{\theta_{0}}{2}+\frac{\pi}{4}\right)} \\
& -\frac{\kappa_{1} \cos \psi_{0}}{2 \cos ^{2}\left(\frac{\theta_{0}}{2}+\frac{\pi}{4}\right)}, \\
\mathcal{M}_{22}= & \kappa_{1} \sin \psi_{0} \operatorname{tg}\left(\frac{\theta_{0}}{2}+\frac{\pi}{4}\right) \\
& -\kappa_{2} \sin \psi_{0} \operatorname{cotg}\left(\frac{\theta_{0}}{2}+\frac{\pi}{4}\right) .
\end{aligned}
$$

An instability arises whenever the real part of either one of the two eigenvalues $\lambda_{1,2}$ of the stability matrix $\mathcal{M}$ becomes positive. Analytical results can be gained by considering the laser operation near the first threshold, i.e., for $J \rightarrow 0$. In this limit, an asymptotic analysis of Eqs. (17)-(23) in the small parameter $J$ can be performed. The calculations show that the real part of one of the two eigenvalues is positive, and its imaginary part is given by

$$
\omega_{\mathrm{Hopf}}=2 \sqrt{\kappa_{1} \kappa_{2}} .
$$

Therefore, for $\kappa_{2} / \kappa_{1} \neq 0$ close to the first laser threshold $J=0$, the stationary $\mathrm{cw}$ mode solution is destabilized via a Hopf (oscillatory) instability, where the frequency of the Hopf instability is given by Eq. (24).

To get an idea of the value of the Hopf instability frequency at first laser threshold and the current level of the second laser threshold, let us consider typical parameters of a semiconductor ring laser with $\alpha=3, \kappa=400 \mathrm{~ns}^{-1}$, and $c=2 s=0.01$ [24]; assuming linear mode couplings $\kappa_{1}=20 \mathrm{~ns}^{-1}$ and $\kappa_{2} / \kappa_{1}=$ 0.1 , the second laser threshold corresponds to a normalized injection current $\mu=1+J_{2} \kappa_{1} /[\kappa(c-s)] \simeq 1.37$, whereas the Hopf instability frequency close to threshold is $\nu_{\mathrm{Hopf}}=$ $(1 / \pi) \sqrt{\kappa_{1} \kappa_{2}} \simeq 2 \mathrm{GHz}$.

We checked the validity of the phase analysis and breakdown of unidirectional emission via a Hopf instability as $\kappa_{2} / \kappa_{1} \neq 0$ by direct numerical simulations of the full laser rate Eqs. (1)-(3), i.e., without adiabatic elimination of the carrier density from the dynamics. As an example, in Fig. 6(a) we show a numerically computed bifurcation diagram of Eqs. (1)-(3) for parameter values $\alpha=3, \kappa=400 \mathrm{~ns}^{-1}, \gamma=0.5 \mathrm{~ns}^{-1}$, $c=2 s=0.01 \quad[24], \quad \kappa_{1}=20 \mathrm{~ns}^{-1}, \quad \kappa_{2} / \kappa_{1}=0.1, \quad$ and $\Theta=0$. According to the phase analysis, for a normalized injection current $\mu>1+J_{2} \kappa_{1} /[\kappa(c-s)] \simeq 1.37$, i.e., above the second laser threshold, stable emission in the $\mathrm{cw}$ mode is observed, whereas for $\mu<1.37$, the laser emission is oscillatory. (a)

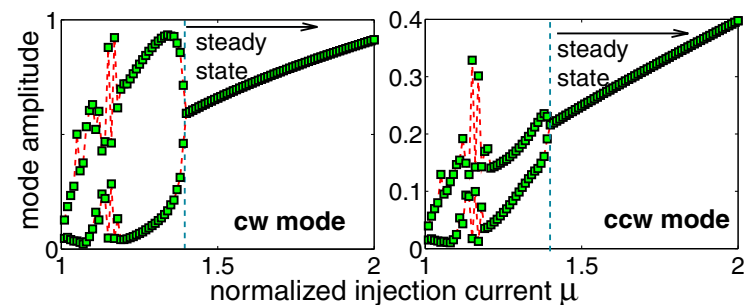

(b)

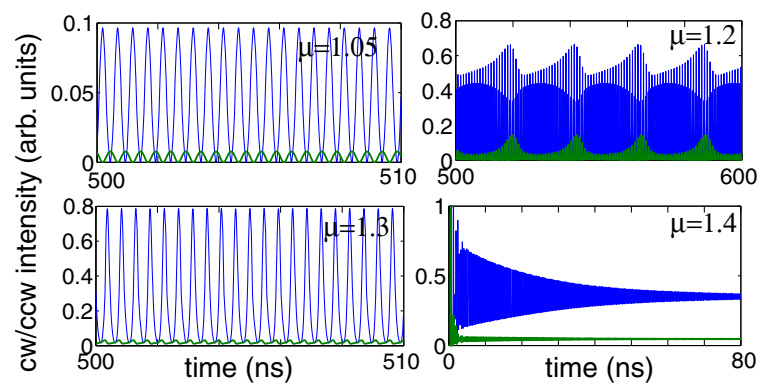

Fig. 6. (a) Numerically computed bifurcation diagram showing the extreme (maxima/minima) of amplitudes $\left|E_{1,2}(t)\right|$ for $\mathrm{cw}$ and $\mathrm{ccw}$ TWMs versus normalized injection current $\mu$. Parameter values are given in the text. For $\mu \gtrsim 1.37$, a stationary regime, corresponding to almost unidirectional emission in the cw mode, is observed, whereas for $\mu \lesssim 1.37$, the dynamics are oscillatory. (b) Numerically computed time evolution of mode intensities $\left|E_{1,2}(t)\right|^{2}$ for cw (thin solid line) and ccw (thick solid line, with lower amplitude) for increasing values of the normalized injection current $\mu$. The laser is switched on at time $t=0$, with the initial condition corresponding to small random amplitudes of $E_{1}$ and $E_{2}$ and $N=0$.

Figure 6(b) shows typical examples of temporal behavior of modal intensities for the cw and ccw TWMs for increasing values of the normalized injection current $\mu$ from the oscillatory $(\mu<1.37)$ to the stationary $(\mu>1.37)$ regimes. Note that close to first laser threshold $(\mu=1.05)$, the mode intensity oscillates in time with a frequency very close to the predicted value of $\nu_{\mathrm{Hopf}} \simeq 2 \mathrm{GHz}$, which is defined by Eq. (24). At higher currents $(\mu=1.2)$, complex oscillatory dynamics are observed until the oscillations are damped and a stable stationary state is found $(\mu=1.4)$ above the "second" laser threshold.

\section{CONCLUSION}

Recent experiments nicely showed that chiral symmetry breaking at an EP provides a viable route for unidirectional laser emission in microlasers [19-21]. By a detailed phase analysis based on a laser rate equation model for a semiconductor microring, we have extended preliminary numerical results reported in previous work [21] and proven that at exact EP unidirectional laser emission is a stable and robust regime and that bistable unidirectional oscillation, corresponding to restoring of the chiral symmetry via nonlinearity, can be observed but at high current levels. Deviations from the EP condition can break unidirectional lasing near threshold via a Hopf instability. However, a "second" laser threshold does exist above which unidirectional stable emission is restored. Our results thus indicate the robustness of unidirectional 
emission based on EP operation and are expected to provide important guidelines for the design of unidirectional microlasers.

Funding. National Science Foundation (NSF) (DMR1506884); Army Research Office (ARO) (W911NF-151-0152).

Acknowledgment. L. F. acknowledges support from $\mathrm{ARO}$ and NSF.

\section{REFERENCES}

1. K. J. Vahala, "Optical microcavities," Nature 424, 839-846 (2003).

2. M. T. Hill, H. J. Dorren, T. De Vries, X. J. Leijtens, J. H. Den Besten, B. Smalbrugge, Y. S. Oei, H. Binsma, G. D. Khoe, and M. K. Smit, "A fast low-power optical memory based on coupled micro-ring lasers," Nature 432, 206-209 (2004).

3. L. Liu, R. Kumar, K. Huybrechts, T. Spuesens, G. Roelkens, E.-J. Geluk, T. de Vries, P. Regreny, D. Van Thourhout, R. Baets, and G. Morthier, "An ultra-small, low-power, all-optical flip-flop memory on a silicon chip," Nat. Photonics 4, 182-187 (2010).

4. M. R. Foreman, J. D. Swaim, and F. Vollmer, "Whispering gallery mode sensors," Adv. Opt. Photon. 7, 168-240 (2015).

5. T. J. Kippenberg, S. M. Spillane, and K. J. Vahala, "Modal coupling in traveling-wave resonators," Opt. Lett. 27, 1669-1671 (2002).

6. M. Sorel, P. J. R. Laybourn, A. Scire, S. Balle, G. Giuliani, R. Miglierina, and S. Donati, "Alternate oscillations in semiconductor ring lasers," Opt. Lett. 27, 1992-1994 (2002).

7. M. Sorel, G. Giuliani, A. Scire, R. Miglierina, S. Donati, and P. J. R. Laybourn, "Operating regimes of GaAs/AIGaAs semiconductor ring lasers: experiment and model," IEEE J. Quantum Electron. 39, 1187-1195 (2003)

8. L. Gelens, S. Beri, G. Van der Sande, G. Mezosi, M. Sorel, J. Danckaert, and G. Verschaffelt, "Exploring multistability in semiconductor ring lasers: theory and experiment," Phys. Rev. Lett. 102, 193904 (2009).

9. L. Gelens, G. Van der Sande, S. Beri, and J. Danckaert, "Phase-space approach to directional switching in semiconductor ring lasers," Phys. Rev. E 79, 016213 (2009).

10. J. P. Hohimer, G. A. Vawter, and D. C. Craft, "Unidirectional operation in a semiconductor ring diode laser," Appl. Phys. Lett. 62, 1185-1187 (1993).

11. J. J. Liang, S. T. Lau, M. H. Leary, and J. M. Ballantyne, "Unidirectional operation of waveguide diode ring lasers," Appl. Phys. Lett. 70, 1192-1194 (1997).
12. G. D. Chern, H. E. Tureci, A. Douglas Stone, R. K. Chang, M. Kneissl, and N. M. Johnson, "Unidirectional lasing from InGaN multiplequantum-well spiral-shaped micropillars," Appl. Phys. Lett. 83, 1710-1712 (2003).

13. J. Wiersig and M. Hentschel, "Combining directional light output and ultralow loss in deformed microdisks," Phys. Rev. Lett. 100, 033901 (2008).

14. Q. J. Wang, C. Yan, N. Yu, J. Unterhinninghofen, J. Wiersig, C. Pflügl, L. Diehl, T. Edamura, M. Yamanishi, H. Kan, and F. Capasso, "Whispering-gallery mode resonators for highly unidirectional laser action," Proc. Natl. Acad. Sci. 107, 22407-22412 (2010).

15. J. Zhu, S. K. Özdemir, L. He, and L. Yang, "Controlled manipulation of mode splitting in an optical microcavity by two Rayleigh scatterers," Opt. Express 18, 23535-23543 (2010).

16. D. Liang, S. Srinivasan, D. A. Fattal, M. Fiorentino, Z. Huang, D. T. Spencer, J. E. Bowers, and R. G. Beausoleil, "Teardrop reflectorassisted unidirectional hybrid silicon microring lasers," IEEE Photon. Technol. Lett. 24, 1988-1990 (2012).

17. B. Redding, L. Ge, Q. Song, J. Wiersig, G. S. Solomon, and H. Cao, "Local chirality of optical resonances in ultrasmall resonators," Phys. Rev. Lett. 108, 253902 (2012)

18. S. Longhi and L. Feng, " $\mathcal{P} \mathcal{T}$-symmetric microring laser-absorber," Opt. Lett. 39, 5026-5029 (2014).

19. M. Kim, K. Kwon, J. Shim, Y. Jung, and K. Yu, "Partially directional microdisk laser with two Rayleigh scatterers," Opt. Lett. 39, 2423-2426 (2014).

20. B. Peng, S. K. Özdemir, M. Liertzer, W. Chen, J. Kramer, H. Yilmaz, J. Wiersig, S. Rotter, and L. Yang, "Chiral modes and directional lasing at exceptional points," Proc. Natl. Acad. Sci. 113, 6845-6850 (2016).

21. P. Miao, Z. Zhang, J. Sun, W. Walasik, S. Longhi, N. M. Litchinitser, and L. Feng, "Orbital angular momentum microlaser," Science 353, 464-467 (2016).

22. W. D. Heiss, "The physics of exceptional points," J. Phys. A 45, 444016 (2012).

23. W. D. Heiss and H. L. Harney, "The chirality of exceptional points," Eur. Phys. J. D 17, 149-151 (2001).

24. G. Van der Sande, L. Gelens, P. Tassin, A. Scire, and J. Danckaert, "Two-dimensional phase-space analysis and bifurcation study of the dynamical behaviour of a semiconductor ring laser," J. Phys. B 41, 095402 (2008).

25. L. Gelens, S. Beri, G. Van der Sande, G. Verschaffelt, and J. Danckaert, "Multistable and excitable behavior in semiconductor ring lasers with broken $Z_{2}$-symmetry," Eur. Phys. J. D 58, 197-207 (2010).

26. H. Zeghlache, P. Mandel, N. B. Abraham, L. M. Hoffer, G. L. Lippi, and T. Mello, "Bidirectional ring laser: stability analysis and timedependent solutions," Phys. Rev. A 37, 470-497 (1988).

27. S. Burkhardt, M. Liertzer, D. O. Krimer, and S. Rotter, "Steady-state ab-initio laser theory for fully or nearly degenerate cavity modes," Phys. Rev. A 92, 013847 (2015). 